This document is the accepted manuscript version of the paper:

JOURNAL OF PHYSICS B: ATOMIC, MOLECULAR AND

OPTICAL PHYSICS 47: Paper: 204023, (2014)

DOI: $10.1088 / 0953-4075 / 47 / 20 / 204023$

\title{
Removing Electrons from More Than One Orbital: Direct and Indirect Ionization to Excited States of Molecular Cations
}

\author{
Arthur Zhao ${ }^{1}$, Péter Sándor ${ }^{1}$, Tamás Rozgonyi ${ }^{2}$ and Thomas Weinacht ${ }^{1}$ \\ ${ }^{1}$ Department of Physics and Astronomy, Stony Brook University, 100 Nicolls Road, Stony Brook, \\ NY 11794-3800, USA \\ ${ }^{2}$ Institute of Materials and Environmental Chemistry, Research Centre for Natural Sciences, \\ Hungarian Academy of Sciences, Budapest 1117, Magyar tudósok körútja 2, Hungary
}

\begin{abstract}
We use velocity map imaging of photoelectrons in coincidence with molecular cations to determine which ionic states are populated via strong field ionisation, and whether the ionisation to excited ionic states proceeds indirectly via the ground ionic state or directly from the neutral. We carry out measurements on a series of molecules that have different energy gaps between the ground ionic state and dissociative excited states. We measure both direct and indirect ionisation to excited states of the molecular cations, and find that the energy gap between non-dissociative and dissociative states plays an important role in determining the amount of excited state ionisation. Direct ionisation to dissociative states is generally comparable to ionization to the ground state for gap energies less than the photon energy, but is suppressed for gap energies larger than the photon energy.
\end{abstract}

\section{Introduction}

There has been significant interest in the study of strong field ionisation (SFI) since it provides access to attosecond electron dynamics via high harmonic generation [1, 2] and allows for direct probing of excited state molecular dynamics on ultrafast timescales with no dark states [3, 4, 5]. While early studies suggested that SFI involved only the most weakly bound electron, recent experiments (in addition to one earlier work [6]) have provided evidence of ionisation from multiple orbitals $[7,8,9,10,11,12,13,14,15,16]$. A detailed study of which orbitals are involved and what final cationic states are populated by SFI is important for understanding the attosecond dynamics underlying SFI, and for using SFI as a tool to follow molecular dynamics.

In this article, we make use of velocity map imaging (VMI) of photoelectrons in coincidence with the corresponding cations [17, 18] in order to determine the amount of ionisation to the ground state vs excited state, and to distinguish between direct and indirect ionisation to excited states. Here we use the term "indirect" to refer to the process whereby the final excited state of the cation is not produced directly from the neutral, but rather via the ground ionic state. We consider a series of molecules and explore the role that the energy difference or gap between the ground and excited ionic 
states plays in determining the amount of ionisation to excited states and whether this ionisation is direct or indirect.

Peaks in the low energy photoelectron spectrum derived from the VMI measurements can be associated with ionisation to different electronic states of the molecular cation [13]. The kinetic energy of electrons ionised to the $i^{\text {th }}$ continuum is given by:

$$
K^{i}=n h \nu-I_{p}^{i}-U_{p}-E_{D S S}^{i}
$$

where $h \nu$ is the energy of a single photon (typically $1.6 \mathrm{eV}$ in these experiments), $I_{p}^{i}$ is the ionisation potential associated with the $i^{\text {th }}$ continuum (i.e. $i^{\text {th }}$ ionic state), $U_{p}$ is the ponderomotive potential and $E_{D S S}^{i}$ is dynamic Stark-shift of the $i^{\text {th }}$ ionic state. As the energy of the photoelectrons is determined at the moment of ionisation, their energy yields information regarding the initial ionic state populated by ionisation, but it does not provide information about whether the molecule is subsequently excited by the laser to a higher lying state of the cation. On the other hand, the fragments produced by the laser-molecule interaction do contain information regarding the final molecular state since the parent ion labels low lying non-dissociative states of the molecule, denoted as $\mathrm{D}_{\mathrm{n}}$ (typically the ground or first excited state, $\mathrm{D}_{0}$ or $\mathrm{D}_{1}$ ), while a fragment ion labels an excited state with sufficient energy to dissociate, denoted as $\mathrm{D}_{\mathrm{d}}$ 田 Thus, by measuring the velocity of the photoelectrons in coincidence with the time-of-flight (ToF) mass spectrum for the ions, we can acquire information about both the initial and final states involved in SFI.

\section{Experimental Apparatus}

We use an amplified Ti:sapphire laser system, producing 30 fs pulses (intensity FWHM) centred at $780 \mathrm{~nm}(1.6 \mathrm{eV})$ with $1 \mathrm{~mJ}$ pulse energy and $1 \mathrm{kHz}$ repetition rate. The focused laser pulses intersect an effusive molecular beam inside our velocity map imaging

spectrometer, all of which is inside a vacuum chamber with a base pressure of $10^{-8}$ Torr. The photoelectrons and photoions are velocity map imaged [20] onto a dual stack of microchannel plates (MCPs) in chevron configuration located about $20 \mathrm{~cm}$ away from the laser focus. The electron shower generated by the MCPs illuminates a phosphor screen behind the MCPs. The voltages on the electrostatic lens are switched in sync with the laser pulse such that both electrons and ions produced from ionisation are collected by the MCPs and the magnitudes of the voltages are adjusted for velocity map imaging - i.e. the velocities of the electrons and ions are mapped to position on the MCPs. For the experiments described here, we are only interested in the time of flight ( ToF) for the ions, which we determined by capacitively coupling to the phosphor screen and directing this signal to a digitizing oscilloscope with a sample rate of $500 \mathrm{Msps}$. Since the imaging

$\ddagger$ We note the additional possibility of dissociation in an ionic state whose minimum energy is below the dissociation threshold by having some fraction of the energy above the ionization potential going to nuclear degrees of freedom rather than photolectron kinetic energy [19]. We considered this possibility for all of the molecules we study, but found it to be relevant to only one of them as noted below. 
process projects a three dimensional electron cloud (with cylindrical symmetry about the laser polarization axis) onto a two dimensional detector, we perform an inverse Abel transformation on the raw image to recover the photoelectron spectrum. Based on the ToF trace, each photoelectron hit can be assigned to a particular cation provided that one and only one electron-ion pair is detected.

Due to limited detection efficiency, false coincidences can occur when two or more molecules are ionised in a given laser shot, and an electron from ionisation of one molecule is detected in coincidence with an ion from a different molecule. In order to minimize the false coincidence rate, we work with a low ionization rate (about 0.6 ionisation events per laser shot), which improves the ratio of true-to-false coincidences. A thorough discussion of false coincidence and a correction algorithm are given in Appendix A. Interested readers are referred to [13] for more details on the experimental apparatus.

\section{Results and Discussion}

We carry out measurements on the following eight molecules: Carbon disulfide $\left(\mathrm{CS}_{2}\right)$, 1,3-Cyclohexadiene $\left(\mathrm{C}_{6} \mathrm{H}_{8}\right), \alpha$ - terpinene $\left(\mathrm{C}_{10} \mathrm{H}_{16}\right)$, 1,3-butadiene $\left(\mathrm{C}_{4} \mathrm{H}_{6}\right)$, Iodobenzene $\left(\mathrm{C}_{6} \mathrm{H}_{5} \mathrm{I}\right)$, Trifluoroiodomethane $\left(\mathrm{CF}_{3} \mathrm{I}\right)$, Bromochloromethane $\left(\mathrm{CH}_{2} \mathrm{BrCl}\right)$ and Iodobromomethane $\left(\mathrm{CH}_{2} \mathrm{IBr}\right)$. These molecules represent a range of sizes and structures, and the energies of their low lying cationic states and dissociation energies are known. The gap energies (defined as the energy difference between $D_{n}$ and $D_{d}$ ) range from below $1 \mathrm{eV}$ to above $4 \mathrm{eV}$, spanning from below to above our photon energy of $1.6 \mathrm{eV}$.

Table 1 gives the IPs and gap energies for the molecules we consider in this study. Wherever possible we used experimental values for the ionic state energies.

Figure 1 shows a cartoon of different ionisation pathways and the photoelectron spectra in coincidence with two different cations from $\mathrm{CH}_{2} \mathrm{BrCl}$. The portions of the spectra in which we are interested are shaded, labelled and linked to their respective ionisation pathways. The photon order indicated is the lowest order consistent with positive photoelectron energy given the ponderomotive shifts estimated based upon our intensity calibration. A detailed discussion of the peak assignments taking into account all of the terms in equation 1 can be found in an earlier manuscript [13].

In order to quantitatively analyse our experimental data, we obtain the absolute yields corresponding to different states by integrating over portions of the spectra in the same fashion as illustrated in this figure, and compute the ratios shown below in table

2. The coincidence photoelectron spectra for all other molecules are shown in Appendix B.

We tried to maintain a constant ionisation rate for all experiments by adjusting the laser pulse intensity. We also worked to achieve a total ionisaton yield of about 0.6 per laser shot in order to obtain a good true coincidence rate (i.e., $\lambda=0.6$ in the notation used in Appendix A. While we were generally able to use a combination of sample pressure and laser intensity to achieve $\lambda=0.6$, it was not possible to achieve 
Table 1: Ionization energies and gap energies for the molecules measured in the experiments sorted by increasing gap energy. $D_{0}$ denotes the ground ionic state and $D_{i}$ denotes the $i^{\text {th }}$ excited ionic state. "(d)" labels dissociative states. Notice that since $\mathrm{CH}_{2} \mathrm{IBr}, \mathrm{C}_{6} \mathrm{H}_{5} \mathrm{I}$ and $\mathrm{CF}_{3} \mathrm{I}$ all have two reasonably resolved peaks in their photoelectron spectra in coincidence with the parent ions (see Figure A1, A2 and A3 in Appendix B), all of them are listed twice in the table.

In case where two states lie too close to each other to be resolved in the photoelectron spectra, we treat them as one state with their average IP:

${ }^{a}$ averaged non-dissociative states' energy;

${ }^{b}$ averaged dissociative states' energy.

\begin{tabular}{|c|c|c|c|c|c|}
\hline Species & $\mathrm{D}_{0}(\mathrm{eV})$ & $\mathrm{D}_{1}(\mathrm{eV})$ & $\mathrm{D}_{2}(\mathrm{eV})$ & $\mathrm{D}_{3}(\mathrm{eV})$ & Gap Energy $(\mathrm{eV})$ \\
\hline $\mathrm{CH}_{2} \mathrm{IBr}[13]$ & 9.69 & 10.26 & $10.91(\mathrm{~d})$ & $11.12(\mathrm{~d})$ & $11.02-10.26=0.76^{b}$ \\
\hline $\mathrm{CH}_{2} \mathrm{BrCl}[13]$ & 10.77 & 11.03 & $11.72(\mathrm{~d})$ & $11.81(\mathrm{~d})$ & $11.76-10.90=0.86^{a}$ \\
\hline $\mathrm{C}_{6} \mathrm{H}_{5} \mathrm{I}[21]$ & 8.75 & 9.45 & 9.74 & $10.55(\mathrm{~d})$ & $10.55-9.60=0.95^{a}$ \\
\hline $\mathrm{CH}_{2} \mathrm{IBr}[13]$ & 9.69 & 10.26 & $10.91(\mathrm{~d})$ & $11.12(\mathrm{~d})$ & $11.02-9.69=1.33^{b}$ \\
\hline $\mathrm{C}_{6} \mathrm{H}_{5} \mathrm{I}[21]$ & 8.75 & 9.45 & 9.74 & $10.55(\mathrm{~d})$ & $10.55-8.75=1.80$ \\
\hline $\mathrm{CF}_{3} \mathrm{I}[22][23][24]$ & 10.37 & 11.09 & $13.02(\mathrm{~d})$ & $15.17(\mathrm{~d})$ & $13.02-11.09=1.93$ \\
\hline $\mathrm{C}_{4} \mathrm{H}_{6}[25]$ & 9.09 & $11.50(\mathrm{~d})$ & $12.44(\mathrm{~d})$ & $\mathrm{n} / \mathrm{a}$ & $11.50-9.09=2.41$ \\
\hline $\mathrm{CF}_{3} \mathrm{I}[22][23][24]$ & 10.37 & 11.09 & $13.02(\mathrm{~d})$ & $15.17(\mathrm{~d})$ & $13.02-10.37=2.65$ \\
\hline $\mathrm{C}_{10} \mathrm{H}_{16}[26]$ & 7.57 & $10.25(\mathrm{~d})$ & $10.71(\mathrm{~d})$ & $\mathrm{n} / \mathrm{a}$ & $10.48-7.57=2.91^{b}$ \\
\hline $\mathrm{C}_{6} \mathrm{H}_{8}[27]$ & 8.25 & $11.67(\mathrm{~d})$ & $13.19(\mathrm{~d})$ & $13.26(\mathrm{~d})$ & $11.67-8.25=3.42$ \\
\hline $\mathrm{CS}_{2}[28]$ & 10.08 & 12.70 & $14.47(\mathrm{~d})$ & $16.19(\mathrm{~d})$ & $14.47-10.08=4.39$ \\
\hline
\end{tabular}

Table 2: Definition of ratios and corresponding figures. The last column indicates how these ratios are obtained from the spectra illustrated in Figure 1. The first ratio $R_{Y i e l d}$ is directly calculated from the total number of photoelectrons measured in coincidence with parent and fragment ions.

\begin{tabular}{|c|c|c|c|}
\hline Ratio & Definition & Plot & Source \\
\hline $\mathrm{R}_{\text {Yield }}$ & $\begin{array}{l}\text { total fragment ion yield } \\
\text { vs. } \\
\text { total ionisation yield }\end{array}$ & Figure 2 & $\begin{array}{c}\text { fragment yield } \\
\text {-to- } \\
\text { (fragment+parent) yield }\end{array}$ \\
\hline $\mathrm{R}_{\mathrm{IF}-\mathrm{P}}$ & $\begin{array}{c}\text { fragment yield via excitation of parent ions } \\
\text { vs. } \\
\text { total parent ion yield }\end{array}$ & Figure 3 & II-to-(I+II) \\
\hline $\mathrm{R}_{\mathrm{DF}-\mathrm{P}}$ & $\begin{array}{l}\text { direct fragment yield } \\
\text { Vs. } \\
\text { total parent ion yield }\end{array}$ & Figure 4 & III-to-(I+II) \\
\hline
\end{tabular}

exactly the same ionisation rate for each molecule. We estimate that the ionisation rate varied between molecules within a factor of two above and below the mean value. Given the strong intensity dependence of the ionisation process, we estimate that this corresponds to variation in the intensity required to generate the same ionisation rate of about 10 percent. We therefore tried to estimate the intensity dependence of the photoelectron spectrum by measuring spectra in coincidence with fragment ions for two different intensities for one of the molecules studied: $\mathrm{CH}_{2} \mathrm{BrCl}$. We used the differences between these spectra taken at different intensities to estimate error bars 


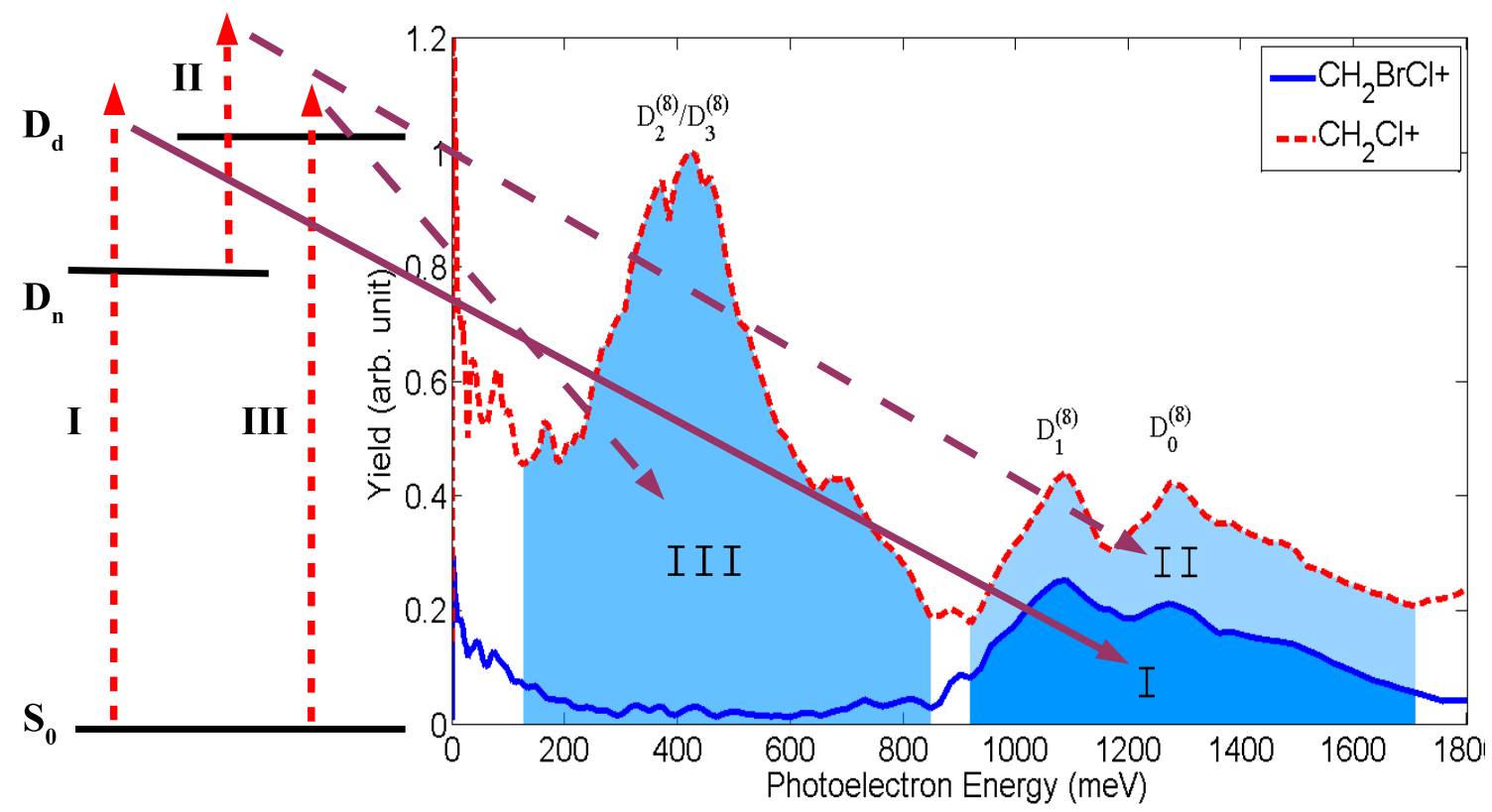

$\mathrm{S}_{0}-$ Ground state

Area I - direct ionization to $\mathrm{D}_{\mathrm{n}}$

$\mathrm{D}_{\mathrm{n}}-$ Non-dissociative ionic (ground) state Area II - indirect ionization to $\mathrm{D}_{\mathrm{d}}$

$\mathrm{D}_{\mathrm{d}}-$ Dissociative ionic excited state

Area III - direct ionization to $\mathrm{D}_{\mathrm{d}}$

Figure 1: Left panel: a diagram illustrating ionisation to two ionic states via three different pathways: "I" represents direct ionisation to the ground ionic state (or another nondissociative ionic state). "II" represents indirect ionisation to a dissociative excited state via the ground state - i.e. ionisation to the ground state followed by a laser driven transition between ionic states. "III"' represents direct ionisation to a dissociative excited state.

Right panel: photoelectron spectrum for the molecule $\mathrm{CH}_{2} \mathrm{BrCl}$ taken in coincidence with the molecular cations shown in the legend. $\mathrm{D}_{\mathrm{i}}^{(\mathrm{j})}$ denotes the $\mathrm{i}^{\text {th }}$ ionic state at the $\mathrm{j}^{\text {th }}$ photon order. Arrows between states and spectra illustrate which ionisation pathways correspond to which features in the photoelectron spectrum. Shading indicates the portions of the photoelectron spectrum which are integrated for calculations of the ratios listed in Table 2 .

for the graphs shown below. In order to check that the uncertainties we estimated based on $\mathrm{CH}_{2} \mathrm{BrCl}$ are reasonable, we measured the photoelectron spectrum (not in coincidence) as a function of intensity for all of the samples, and found that for small variations in intensity (near the values used for the coincidence measurements) there was only variation in the total yield and no significant variation in the shape of the spectra. Thus, we believe the relative uncertainty obtained from $\mathrm{CH}_{2} \mathrm{BrCl}$ provides a reasonable estimate of the uncertainty for the other samples. The error bars in Figure 3 and 4 are obtained in this fashion.

Since the efficiency of the MCPs varies with mass for a given energy [29, 30], we have corrected our measured photoelectron spectra with the detection efficiencies extrapolated from Fig. 3 in [30]. The details of the correction algorithm are given in Appendix A 


\subsection{Fraction of fragment ions}

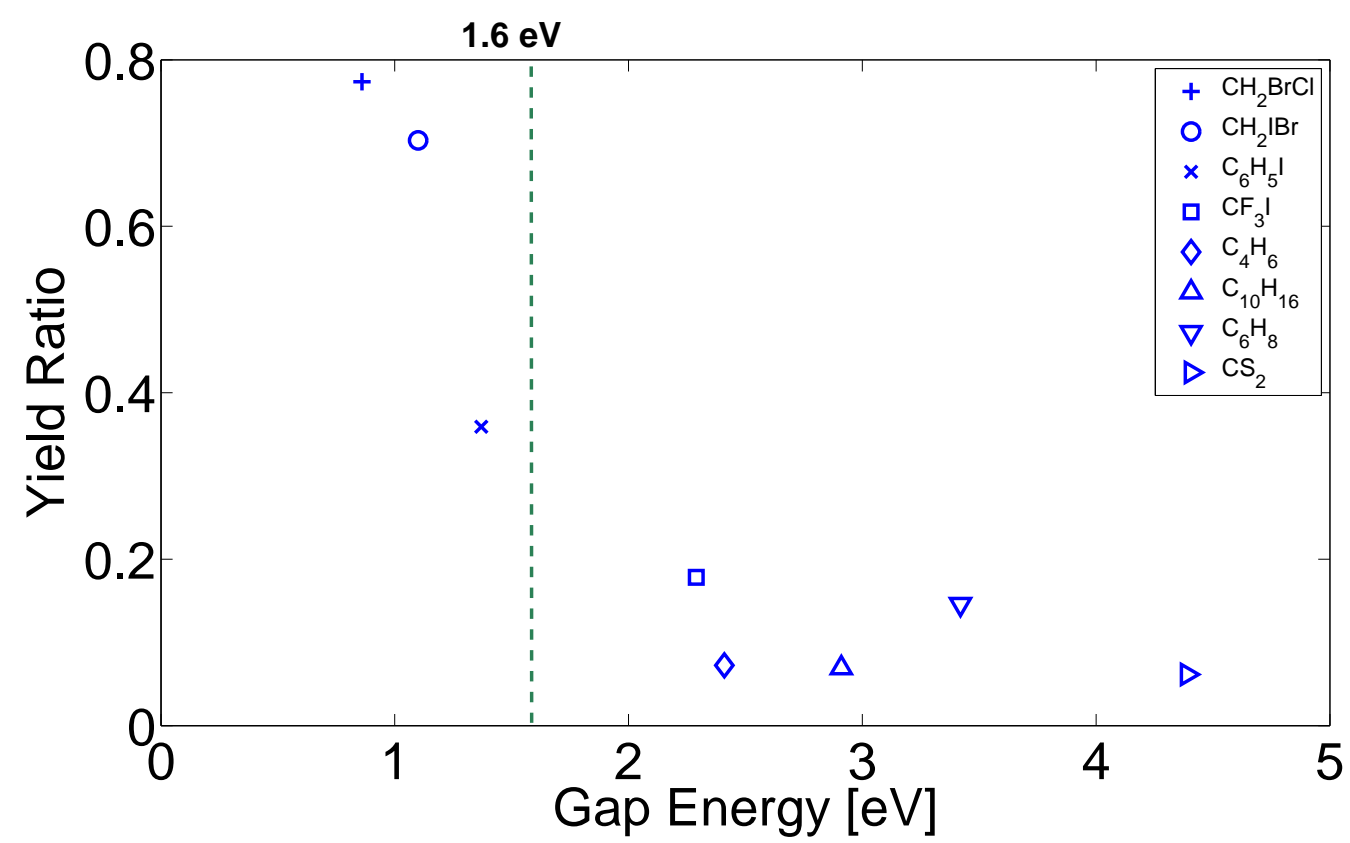

Figure 2: $\mathrm{R}_{\text {Yield }}$, total fragment ion yield divided by the total ionisation yield. The ratios are calculated directly from the numbers of photoelectrons collected in coincidence with the parent and fragment ions. Here the fragment production includes both direct and indirect pathways.

Now we examine the ratios listed in Table 2 . The first ratio $R_{\text {Yield }}$ plotted in Figure 2 shows the fraction of the ionisation that is dissociative (i.e. the ratio of photoelectrons in coincidence with fragments to the total photoelectron yield). Since $\mathrm{CH}_{2} \mathrm{IBr}, \mathrm{C}_{6} \mathrm{H}_{5} \mathrm{I}$ and $\mathrm{CF}_{3} \mathrm{I}$ all have two energy gaps listed in Table 1, in this plot we use their average values: $1.10 \mathrm{eV}$ for $\mathrm{CH}_{2} \mathrm{IBr}, 1.37 \mathrm{eV}$ for $\mathrm{C}_{6} \mathrm{H}_{5} \mathrm{I}$ and $2.29 \mathrm{eV}$ for $\mathrm{CF}_{3} \mathrm{I}$. The measurement shows a trend of decreasing ionization to excited states with increasing gap size, which is reasonable given the highly nonlinear dependence of the ionization rate on laser intensity. We note that the photon energy $(1.6 \mathrm{eV})$ roughly divides the molecules into ones which have a significant dissociative ionization yield and ones which don't.

\subsection{Indirect fragment vs parent}

$\mathrm{R}_{\mathrm{IF}-\mathrm{P}}$, plotted in Figure 3 shows the fraction of ionisation to non-dissociative which is followed by further excitation in the laser field to dissociative states, as a function of the gap energy. Direct ionisation to dissociative states is excluded here. While there is clearly a trend of decreasing indirect ionisation with gap energy, there is also a significant spread of ratios around each gap energy. Earlier work has noted the importance of ionic resonances in determining whether dissociation occurs in SFI [31, 32, 33, 34]. While the energy separations and transition dipole moments between ionic states at the Franck Condon (FC) point for vertical ionization are important, vibrational dynamics during the ionisation process can also lead to dynamic resonances and transitions between ionic 


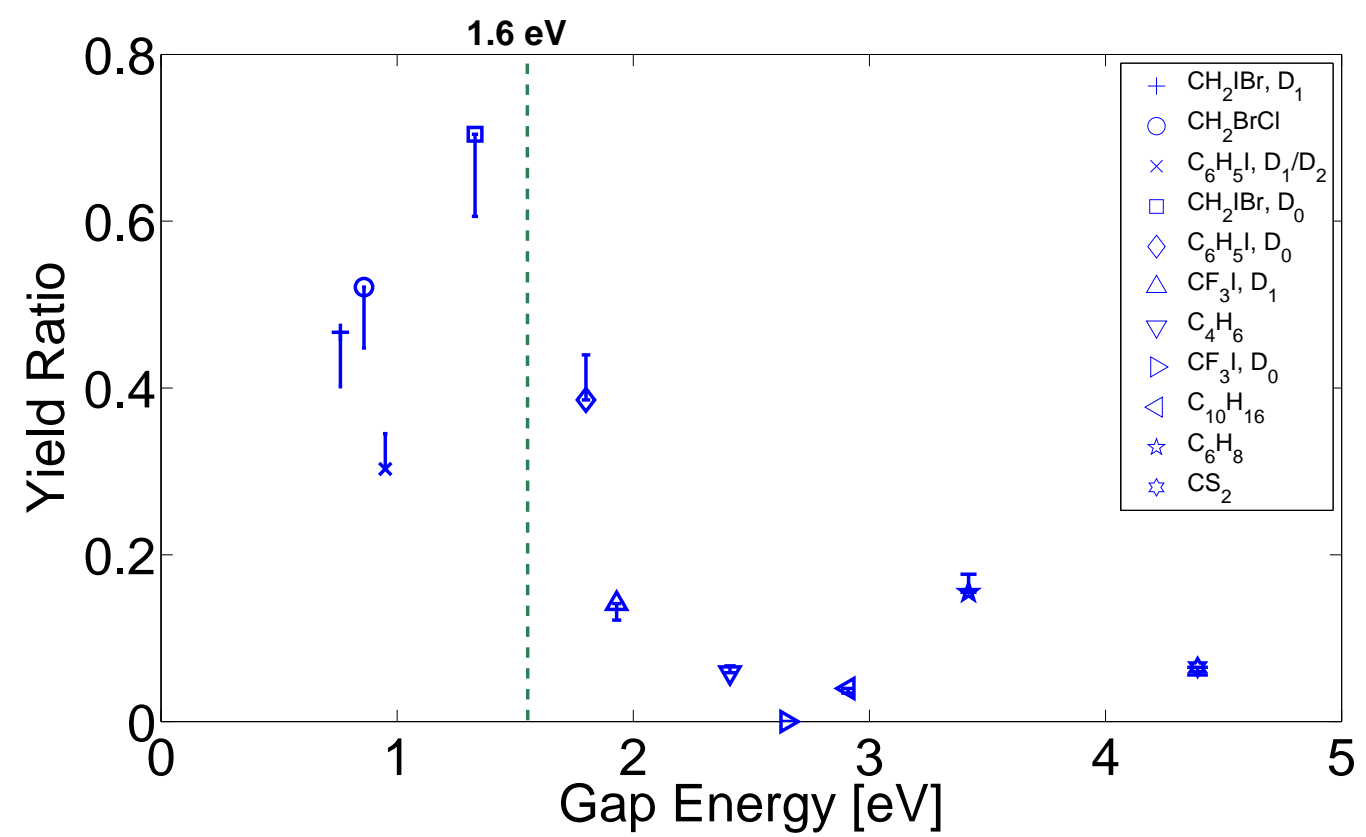

Figure 3: $R_{\mathrm{IF}-\mathrm{P}}$, fragment production from excitation of parent ion divided by total parent ion production. This shows the fraction of the initially produced parent ions that are further excited to dissociative states. The ratios are calculated by integrating relevant regions in the Photoelectron spectrum measured in coincidence with the parent and fragment ions. The choice of these regions is illustrated in Figure 1, in this case, the ratio between Area II and (Area I + Area II).

states away from the FC point [35]. Furthermore, multiphoton absorption can also lead to transfer between ionic states [36].

In order to illustrate how the various factors (resonance conditions, vibrational dynamics and transition dipole moments) can influence the extent of indirect ionization, we compare two of the molecular samples we considered in more detail. For $\mathrm{CH}_{2} \mathrm{IBr}$, we measure substantial indirect ionization from $\mathrm{D}_{0}$ despite the fact that there is no resonance at the $\mathrm{FC}$ point. However, motion along the $\mathrm{D}_{0}$ potential energy surface can lead to a resonance between $\mathrm{D}_{0}$ and $\mathrm{D}_{2} / \mathrm{D}_{3}$ during the laser pulse [36]. Since the transition dipole moments between these states is large, population can be efficiently transferred to $\mathrm{D}_{2} / \mathrm{D}_{3}$ during the tail of the ionization pulse, leading to the large indirect ionization yield observed. In contrast, we observe no indirect ionization to $\mathrm{D}_{2}$ (the first dissociative state) from $\mathrm{D}_{0}$ in $\mathrm{CF}_{3} \mathrm{I}$ (see the data point at around $2.6 \mathrm{eV}$ as well as Figure A3). This can be explained by the vanishing transition dipole moment between these states (calculated at the same level of theory as in earlier work [37, 13]) and the fact that the states are separated by an energy much larger than the photon energy, even if one considers vibrational motion along $\mathrm{D}_{0}$.

\subsection{Direct fragment vs parent}

$\mathrm{R}_{\mathrm{DF}-\mathrm{P}}$ plotted in Figure 4 compares two competing ionisation processes from the neutral: direct removal of an electron from a high-lying vs more deeply bound orbital. The ratio 


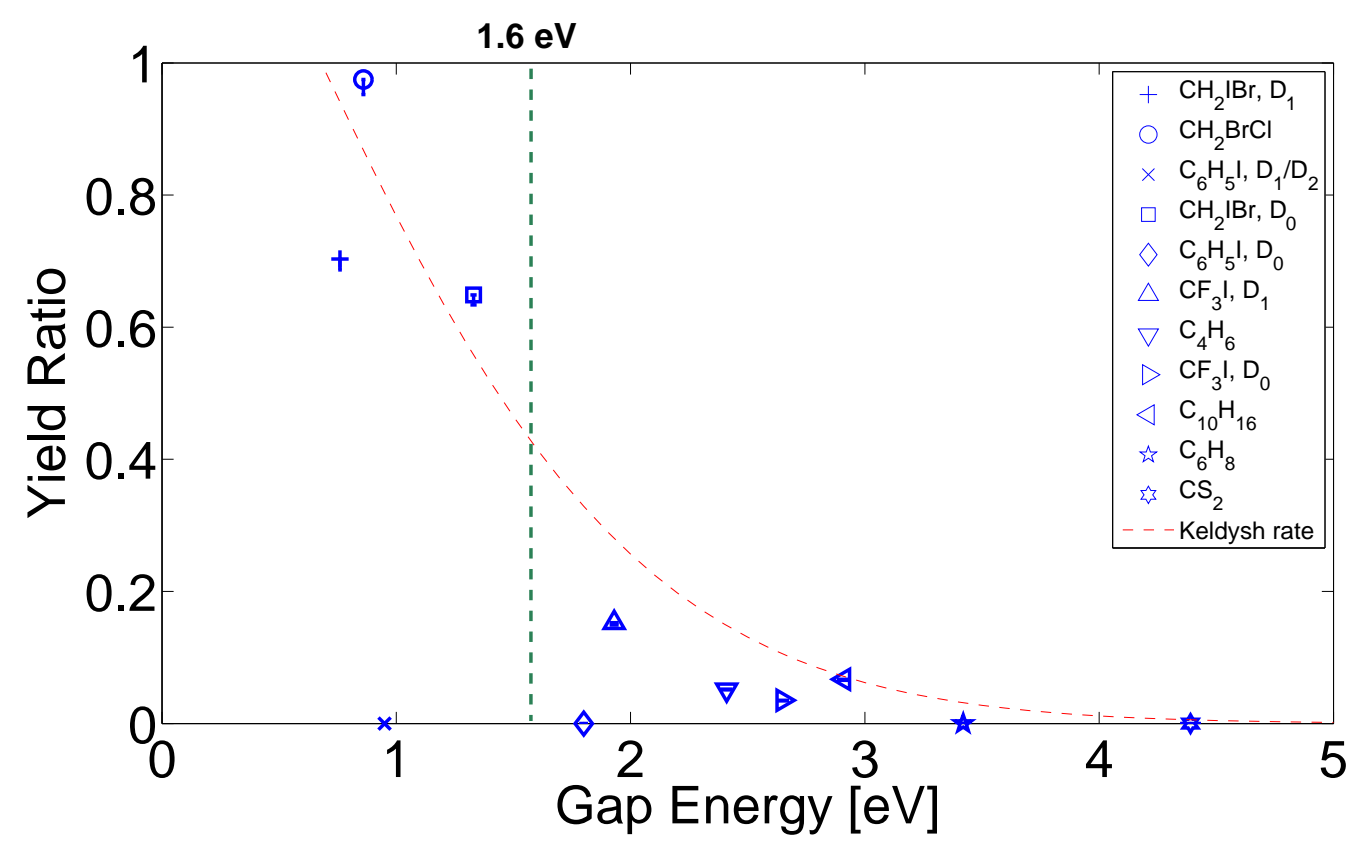

Figure 4: $R_{\mathrm{DF}-\mathrm{P}}$, direct fragment production divided by total parent ion production. The latter includes all the population reaching non-dissociative states, whether staying there in the end or excited further to dissociative states. This figure compares the likelihoods of ionising a high-lying and a lowlying orbital electron. The ratios are calculated by integrating relevant regions in the photoelectron spectra measured in coincidence with the parent and fragment ions. The choice of these regions is illustrated in Figure 1, in this case, the ratio between Area III and (Area I + Area II).

shows a strong dependence on the gap energy, especially near the photon energy of $1.6 \mathrm{eV}$, where an abrupt jump can been seen. A natural framework for interpreting the measurements is provided by the strong field approximation (SFA). In the SFA, the ionisation rate is proportional to the product of the Dyson norm and the Keldysh rate (Eq. 21 in [14]). The Dyson norm $\left\langle\phi_{l m} \mid \phi_{l m}\right\rangle$ is simply the norm of the Dyson orbital $\mid \phi_{l m}>$, which is constructed as the overlap between the neutral $|l\rangle$ and ionic states $\mid m>$. The Keldysh rate results from the calculation of tunnel ionisation and is dominated by an exponential term $e^{-\mathrm{IP}}$, where IP is the ionisation potential. Considering low-lying ionic states here, one expects the Dyson norms to be all close to unity, since the low lying ionic states are dominated by single hole configurations, corresponding to removing an electron from a single orbital. Considering now the dependence on the IP, we focus on two ionic states: a non-dissociative state $a$ with IP $E_{a}$ and a dissociative state $b$ with IP $E_{b}$. According to the SFA, the ionisation rates to these two states are proportional to $e^{-E_{a}}$ and $e^{-E_{b}}$ and it follows that the ratio of their yields is simply given by $e^{-\left(E_{b}-E_{a}\right)} . E_{b}-E_{a}$ is nothing but the gap energy defined earlier, and the ratio is plotted in Figure 4. The red dashed curve is the Keldysh rate. We note that while this curve captures the general decreasing trend of the data, there is a sharp drop in the measured ratio at roughly the photon energy. Furthermore, a few of the molecular species, notably iodobenzene $\left(\mathrm{C}_{6} \mathrm{H}_{5} \mathrm{I}\right)$ deviate significantly from the curve. 
Indeed, $\mathrm{C}_{6} \mathrm{H}_{5} \mathrm{I}, \mathrm{C}_{6} \mathrm{H}_{8}$ and $\mathrm{CS}_{2}$ show no significant direct ionisation to excited dissociative states. This suggests that there are deviations in the strong field ionization to excited states from SFA predictions, consistent with earlier measurements and calculations [14].

As one can see, both Figure 3 and 4 show a trend of decreasing yields to dissociative states relative to the ground (or first excited) ionic state. This indicates that as the energy gap gets wider, it becomes less likely to ionise directly to a dissociative state or for a low-lying ionic state to absorb additional photon(s) in order to be excited to a dissociative state. The photon energy $(1.6 \mathrm{eV})$ appears to be an important threshold in determining the amount of ionisation to excited states.

\section{Conclusion}

In conclusion, we have measured photoelectron spectra in coincidence with molecular fragment cations resulting from strong field ionisation of eight different molecules and compared the ionisation yield to different ionic states. We find both direct and indirect ionisation to excited states of the molecular cations, and find that the energy gap between non-dissociative and dissociative states plays an important role in determining the amount of excited state ionisation. Direct ionisation to dissociative states is generally comparable to ionisation to the ground state for gap energies less than the photon energy, but is significantly suppressed if the gap energy is larger than the photon energy. Indirect ionisation to dissociative states depends further on the transition dipole moments between cationic states and the variation in energy with nuclear coordinates. The authors gratefully acknowledge support from the National Science Foundation under award number 1205397. 


\section{Appendix A - Probability of Coincidence}

Here we provide a detailed assessment of true vs false coincidence rates given the finite detection efficiency of our detector. Our measurements consist of velocity map imaging measurements of the photoelectrons in coincidence with time-of-flight(ToF) measurements of the photoions. The data collection algorithm accepts a data point if and only if it detects exactly one photoion peak in the ToF trace and exactly one photoelectron hit in the image. However, due to limited $(<1)$ detection efficiency, it is possible that a measured electron and fragment ion pair come from different molecules. We distinguish two cases:

(1) Valid coincidence: this includes "true coincidence" - the electron and ion come from the same molecule, and "cross coincidence" the electron and ion come from two different molecules which, however, result in the same ionic state) .

(2) False coincidence: the electron and ion come from two separate molecules which end up in different ionic states.

To calculate the occurrence of each case and compare them, we first assume the

reaction probability follows a Poisson distribution $\frac{\lambda^{k} e^{-\lambda}}{k !}$, with $\lambda$ being the expected ionisation occurrence per laser shot ( $\approx 0.6$ in our experiment). Most of our measurements show one dominant fragment ion production along with the parent ion. In cases where there are multiple competing fragment ions, we found that the different fragments typically have similar photoelectron spectrum, and therefore we combine their photoelectron spectrum for better statistics. In light of this and to simplify the derivation, we denote by $b_{p}$ and $b_{c}$ the branching ratios of the parent and the fragment ion, respectively, with $b_{p}+b_{c}=1$. We also denote by $\eta_{p}$ and $\eta_{c}$ the detection efficiencies for the parent and fragment ion, respectively, with $\eta_{e}$ being the detection efficiency for photoelectrons.

\section{Probability of Valid Coincidence}

$$
\begin{aligned}
& V\left(\lambda, b_{p}, \eta_{p}, \eta_{c}, \eta_{e}\right) \\
= & \sum_{k=1}^{\infty} \frac{\lambda^{k} e^{-\lambda}}{k !} \sum_{j=1}^{k} \frac{k !}{j !(k-j) !} b_{p}^{j}\left(1-b_{p}\right)^{k-j}\left(1-\left(1-\eta_{p}\right)^{j}\right)\left(1-\eta_{c}\right)^{k-j} j \eta_{e}\left(1-\eta_{e}\right)^{k-1} \\
= & e^{-\lambda} \lambda b_{p} \eta_{e}\left[e^{\lambda\left(1-\eta_{e}\right)\left(1-\eta_{c}+b_{p} \eta_{c}\right)}-\left(1-\eta_{p}\right) e^{\lambda\left(1-\eta_{e}\right)\left(1-\eta_{c}+b_{p} \eta_{c}-b_{p} \eta_{p}\right)}\right]
\end{aligned}
$$

This result can be readily generalised for multiple fragment ion species with various branching ratios and detection efficiencies. §

$\S$ However, this is much more complex and not necessary. To deal with multiple species, it's easier to treat them as two species - the spectrum of one species and the sum of the spectra of the rest. The detection efficiency can be renormalised using the branching ratio, ie, a weighted average. 


\section{Probability of False Coincidence}

Now we consider the case that the detected photoion-electron pair come from two molecules which produce different photoion fragments. The probability of false coincidence in the parent due to the fragment (i.e. a photoelectron which came from a molecule that fragmented, but is measured in coincidence with a parent ion from a different molecule) is given by:

$$
\begin{aligned}
& F\left(\lambda, b_{p}, \eta_{p}, \eta_{c}, \eta_{e}\right) \\
= & \Sigma_{k=1}^{\infty} \frac{\lambda^{k} e^{-\lambda}}{k !} \Sigma_{j=1}^{k} \frac{k !}{j !(k-j) !} b_{p}^{j}\left(1-b_{p}\right)^{k-j}\left(1-\left(1-\eta_{p}\right)^{j}\right)\left(1-\eta_{c}\right)^{k-j}(k-j) \eta_{e}\left(1-\eta_{e}\right)^{k-1} \\
= & e^{-\lambda} \eta_{e} \lambda\left(1-b_{p}\right)\left(1-\eta_{c}\right)\left[e^{\lambda\left(1-\eta_{c}+b_{p} \eta_{c}\right)}-e^{\lambda\left(1-\eta_{c}+b_{p} \eta_{c}-b_{p} \eta_{p}\right)}\right]
\end{aligned}
$$

\section{Correction to Systematic Error}

False coincidences introduce a systematic error in the Photoelectron spectrum. Here we try to make a first-order correction. We write the spectra as follows:

$$
\begin{aligned}
& m_{p}(E)=V_{p}\left(\lambda, b_{p}\right) p(E)+F_{p}\left(\lambda, b_{p}, b_{c}\right) c(E) \\
& m_{c}(E)=V_{c}\left(\lambda, b_{c}\right) c(E)+F_{c}\left(\lambda, b_{c}, b_{p}\right) p(E)
\end{aligned}
$$

where $m_{p}(E), m_{c}(E)$ are the measured photoelectron spectra for the parent and fragment ions, respectively, $E$ denotes the photoelectron energy, and $p(E), c(E)$ are the real photoelectron spectra for the parent and fragment, respectively. Both are normalized such that the integral over all energies is 1 :

$$
\int p(E) d E=\int c(E) d E=1
$$

$V_{p}, V_{c}$ are the probabilities of a valid coincidence for the parent and fragment, respectively. $F_{p}, F_{c}$ are the probabilities of false coincidence for the parent and fragment, respectively. $b_{p}$ is calculated from the measured spectra:

$$
b_{p}=\left(\int m_{p} d E\right)\left(\int\left(m_{p}+m_{c}\right) d E\right)^{-1}, \quad b_{c}=1-b_{p}
$$

Solving the system of equations A.5 above, we get

$$
p(E)=\frac{m_{p} V_{c}-m_{c} F_{p}}{V_{p} V_{c}-F_{p} F_{c}} \quad c(E)=\frac{m_{c} V_{p}-m_{p} F_{c}}{V_{c} V_{p}-F_{c} F_{p}}
$$

This is not the complete correction since $b_{p}$ is obtained from the measured spectra, not the real ones. Taking into account the branching ratios, the final photoelectron spectra are : $p(E) b_{p}$ and $c(E) b_{c}$. One can iterate this process by re-calculating $b_{p}$ using the resultant spectra to get a better correction. 


\section{Numerical Results}

Here we give the numerical values for $\frac{V_{p}}{V_{p}+F_{p}}$ and $\frac{V_{c}}{V_{c}+F_{c}}$, i.e., the fraction of valid coincidences, for all samples. The first number in the table is obtained from A.2 and A.4 while the second number is obtained from a separate Monte Carlo simulation. The two approaches yield consistent results.

Table A1: Fraction of valid coincidences

\begin{tabular}{|c|c|c|c|c|c|c|c|c|}
\hline Species & $\mathrm{CS}_{2}$ & $\mathrm{C}_{6} \mathrm{H}_{8}$ & $\mathrm{C}_{10} \mathrm{H}_{16}$ & $\mathrm{C}_{4} \mathrm{H}_{6}$ & $\mathrm{C}_{6} \mathrm{H}_{5} \mathrm{I}$ & $\mathrm{CF}_{3} \mathrm{I}$ & $\mathrm{CH}_{2} \mathrm{BrCl}$ & $\mathrm{CH}_{2} \mathrm{IBr}$ \\
\hline$\frac{V_{p}}{V_{p}+F_{p}}$ & 0.99 & 0.98 & 0.99 & 0.99 & 0.93 & 0.95 & 0.89 & 0.89 \\
\hline$\frac{V_{c}}{V_{c}+F_{c}}$ & 0.86 & 0.98 & 0.99 & 0.99 & 0.94 & 0.96 & 0.87 & 0.89 \\
\hline
\end{tabular}




\section{Appendix B - Photoelectron spectrum of Selected Molecules}

$\mathrm{D}_{\mathrm{i}}^{(\mathrm{j})}$ denotes the $\mathrm{i}^{\text {th }}$ ionic state at the $\mathrm{j}^{\text {th }}$ photon order, with $\mathrm{i}=0$ corresponding to the ground state. $\mathrm{CH}_{2} \mathrm{IBr}, \mathrm{C}_{6} \mathrm{H}_{5} \mathrm{I}$ and $\mathrm{CF}_{3} \mathrm{I}$ all contribute two data points each in the ratio figures. When the relevant peaks overlap, we try to fit multiple Gaussians to them to have a better discrimination. However, a comparison between integrating over a Gaussian fit and a straight-cut region in the case of $\mathrm{CH}_{2} \mathrm{IBr}$ shows little discrepancy in the resultant ratios, that is, it doesn't affect the trend observed in the ratio plots. So we only carry out a Gaussian fit when it's necessary. For Figure A4 and A5, the spectra in coincidence with the fragments are rather flat and have very low yields, which is something we don't quite understand yet. In these cases, the assignment of the ionic states and the choice of the integration regions are based on both the ionic state energies and the comparison with the spectra in coincidence with the parent. More specifically, in Figure A4 and A5, the light green and dark blue regions of the spectra in coincidence with the fragment ions have similar yields while the spectra in coincidence with the parent ions vary by a lot. Hence we believe the green regions are direct ionisation to dissociative states.

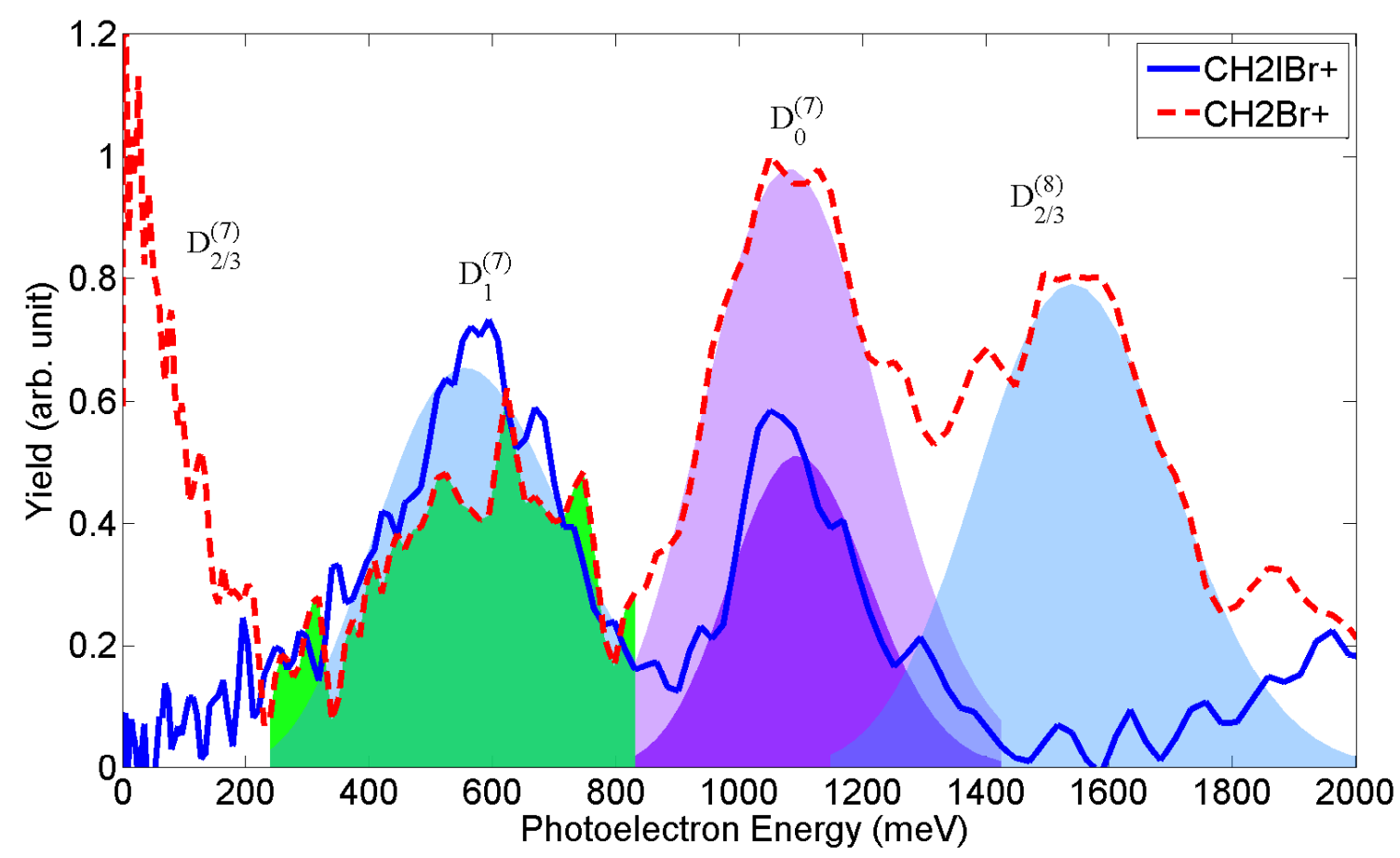

Figure A1: Photoelectron spectrum of $\mathrm{CH}_{2} \mathrm{IBr}$.

A multi-Gaussian fit is carried out and the ratio calculations are based on the shaded areas indicated in the figure because there are significant overlaps between the peaks in the photoelectron spectrum. We note that in an earlier study of the molecule $\mathrm{CH}_{2} \mathrm{IBr}$ [37, we found negligible indirect ionisation yield to $\mathrm{D}_{2} / \mathrm{D}_{3}$ at low laser intensity based on an indirect pump-probe analysis and theoretical calculations. We feel that our current results, which find significant indirect ionisation to $D_{2} / D_{3}$, provide an improved measurement since the photoelectron spectrum gives a more direct indication of the state resolved ionisation yields. 


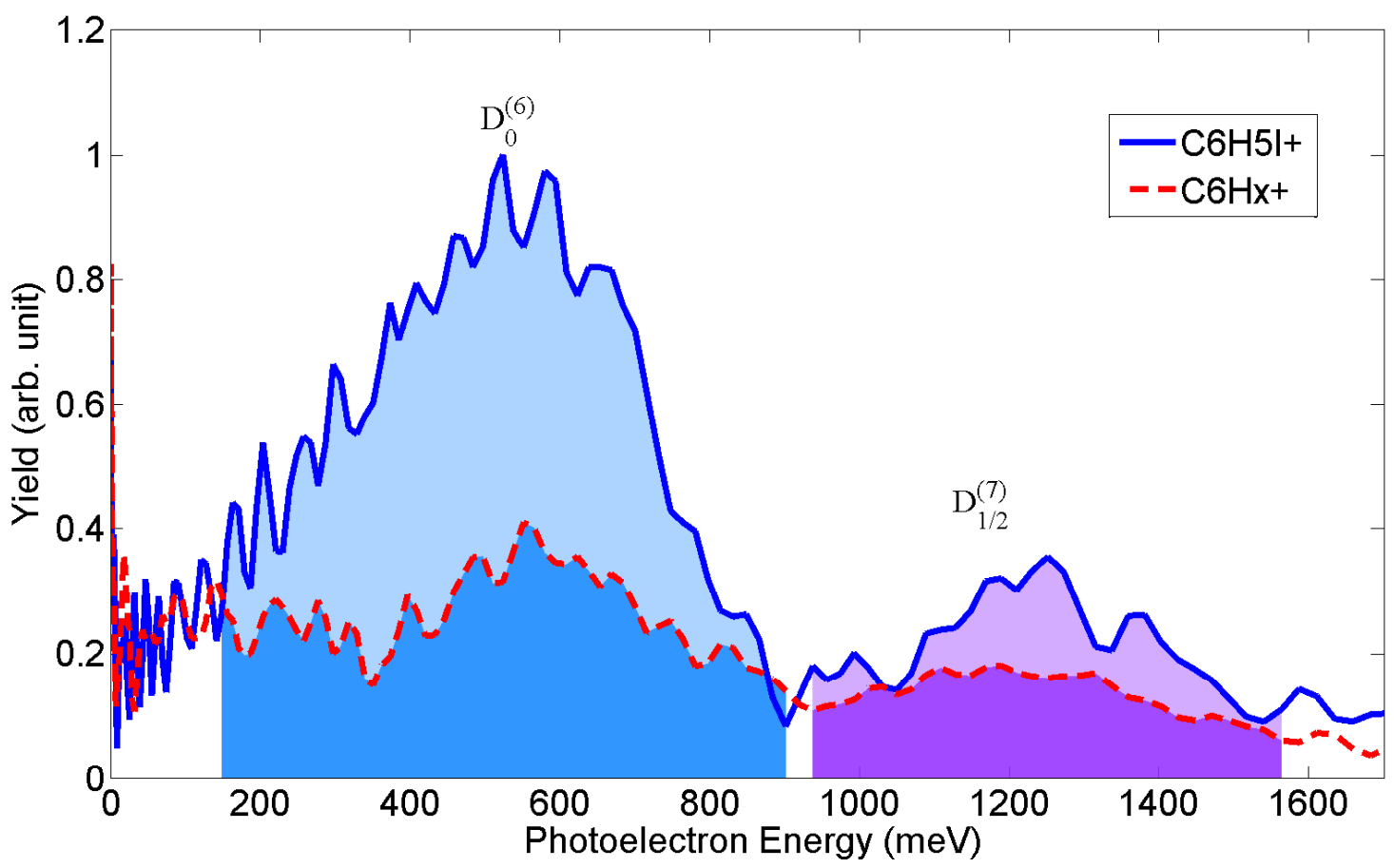

Figure A2: Photoelectron spectrum of $\mathrm{C}_{6} \mathrm{H}_{5} \mathrm{I}$.

No direct ionisation to dissociative states is observed. 


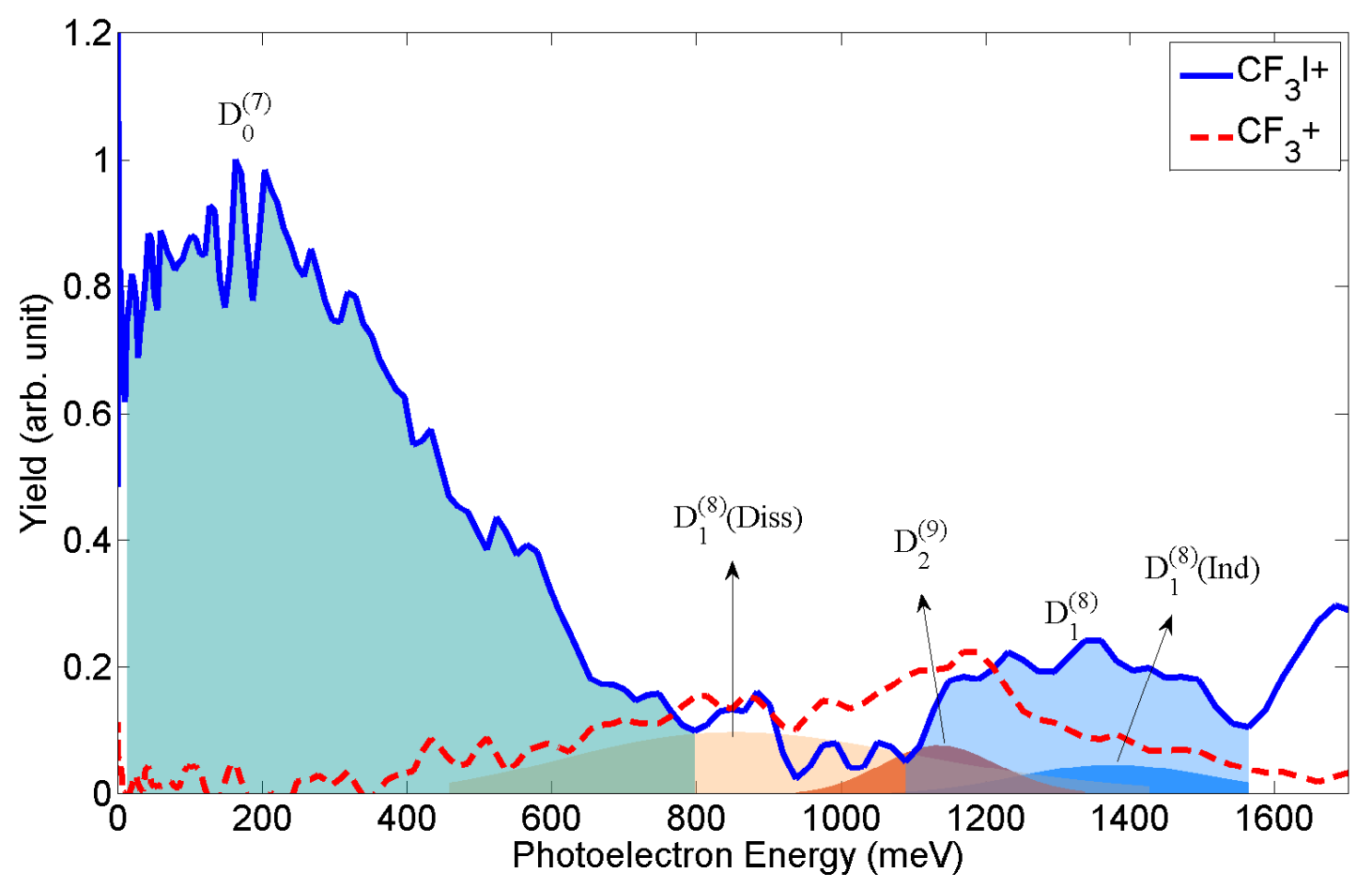

Figure A3: Photoelectron spectrum of $\mathrm{CF}_{3} \mathrm{I}$.

Although state $\mathrm{D}_{1}$ itself is non-dissociative, it is possible to ionise the molecule to a vibrationally excited $\mathrm{D}_{1}$ which may dissociate due the extra energy available from vibrational degrees of freedom. We label this feature as $\mathrm{D}_{1}^{(8)}$ (Diss). This is also observed in [19. It is also worth noting that there is no post-ionisation excitation from the state $\mathrm{D}_{0}$, in contrast to all other molecules. We believe this is due to a negligible coupling strength and a large energy gap between $\mathrm{D}_{0}$ and excited ionic states. A Gaussian fit is applied to discriminate various features in the spectra. First, we fit a Gaussian curve to $\mathrm{D}_{1}^{(8)}(\mathrm{Ind})$, shaded in dark blue, such that its shape resemble that of $\mathrm{D}_{1}^{(8)}$, shaded in light blue, and it's below the red spectrum. Then we subtract this Gaussian from the red spectrum and fit the resultant spectrum with two Gaussian curves, which are labelled $D_{1}^{(8)}($ Diss $)$ and $D_{2}^{(9)}$. The assignment of these peaks are base on the ionic state energies and the discussion in [19]. 


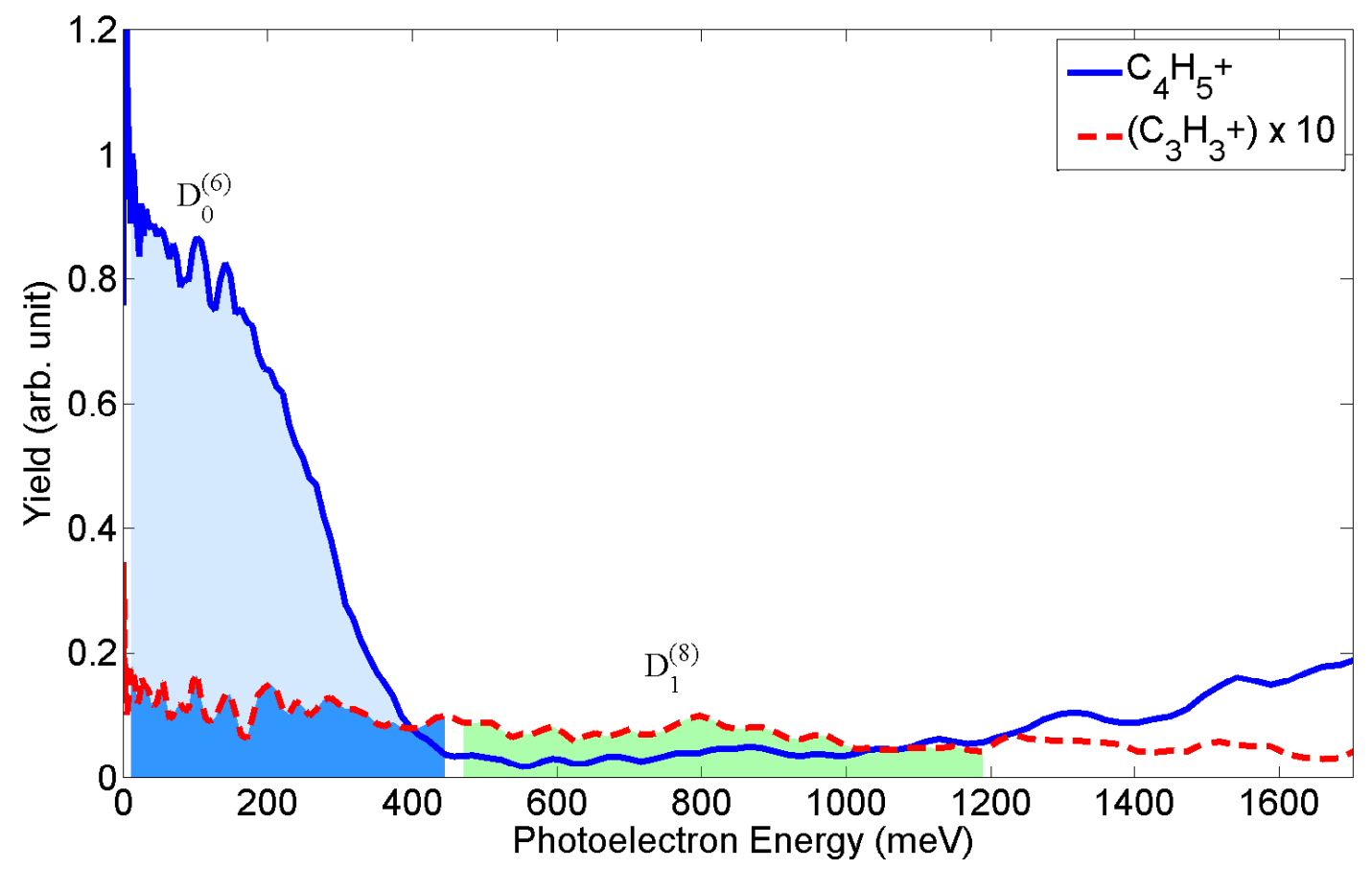

Figure A4: Photoelectron spectrum of $\mathrm{C}_{4} \mathrm{H}_{6}$.

The spectrum in coincidence with the fragment ion is multiplied by 10 for better viewing.

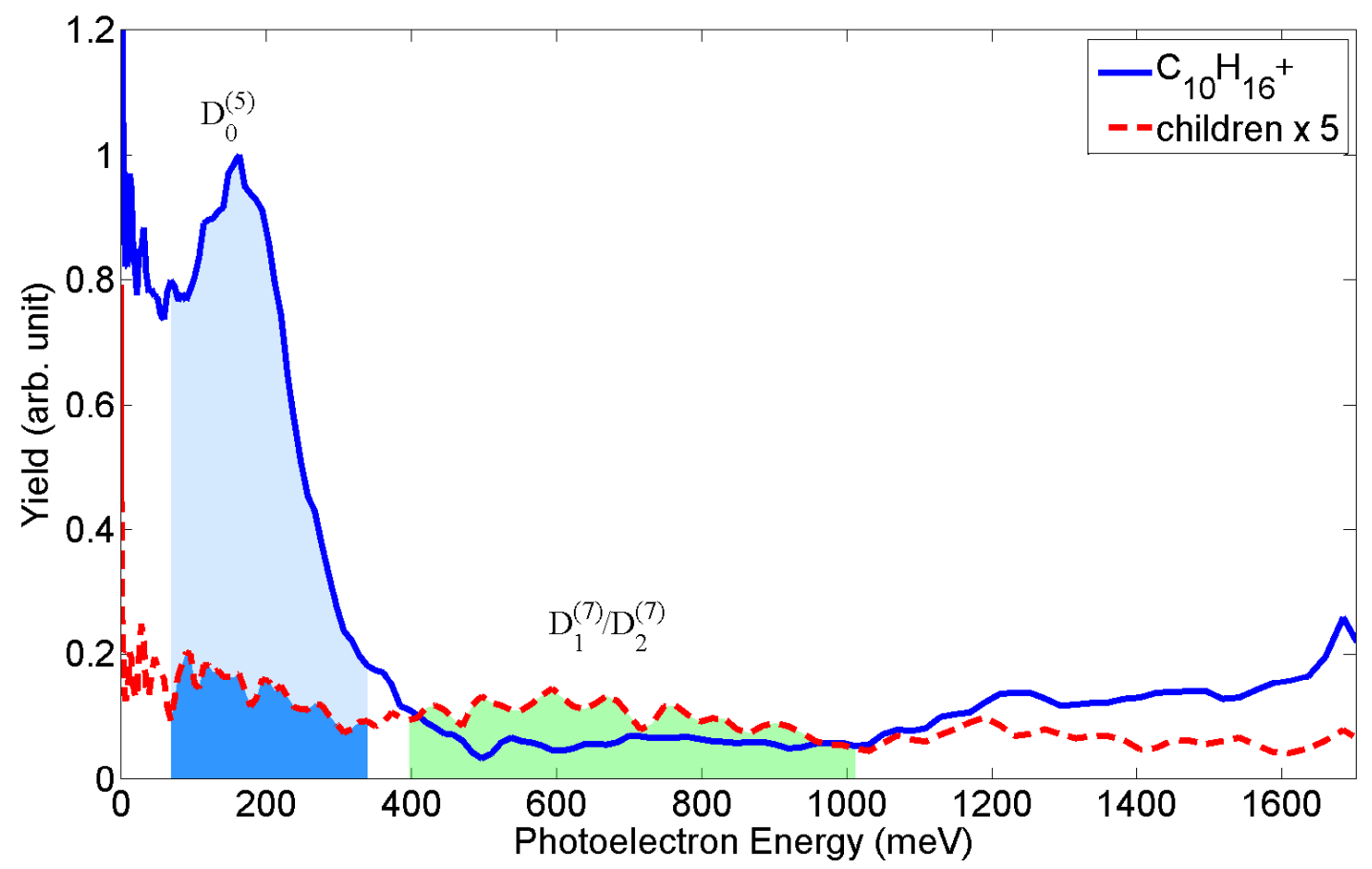

Figure A5: Photoelectron spectrum of $\mathrm{C}_{10} \mathrm{H}_{16}$.

The spectrum in coincidence with the fragment ions is multiplied by 5 for better viewing. 


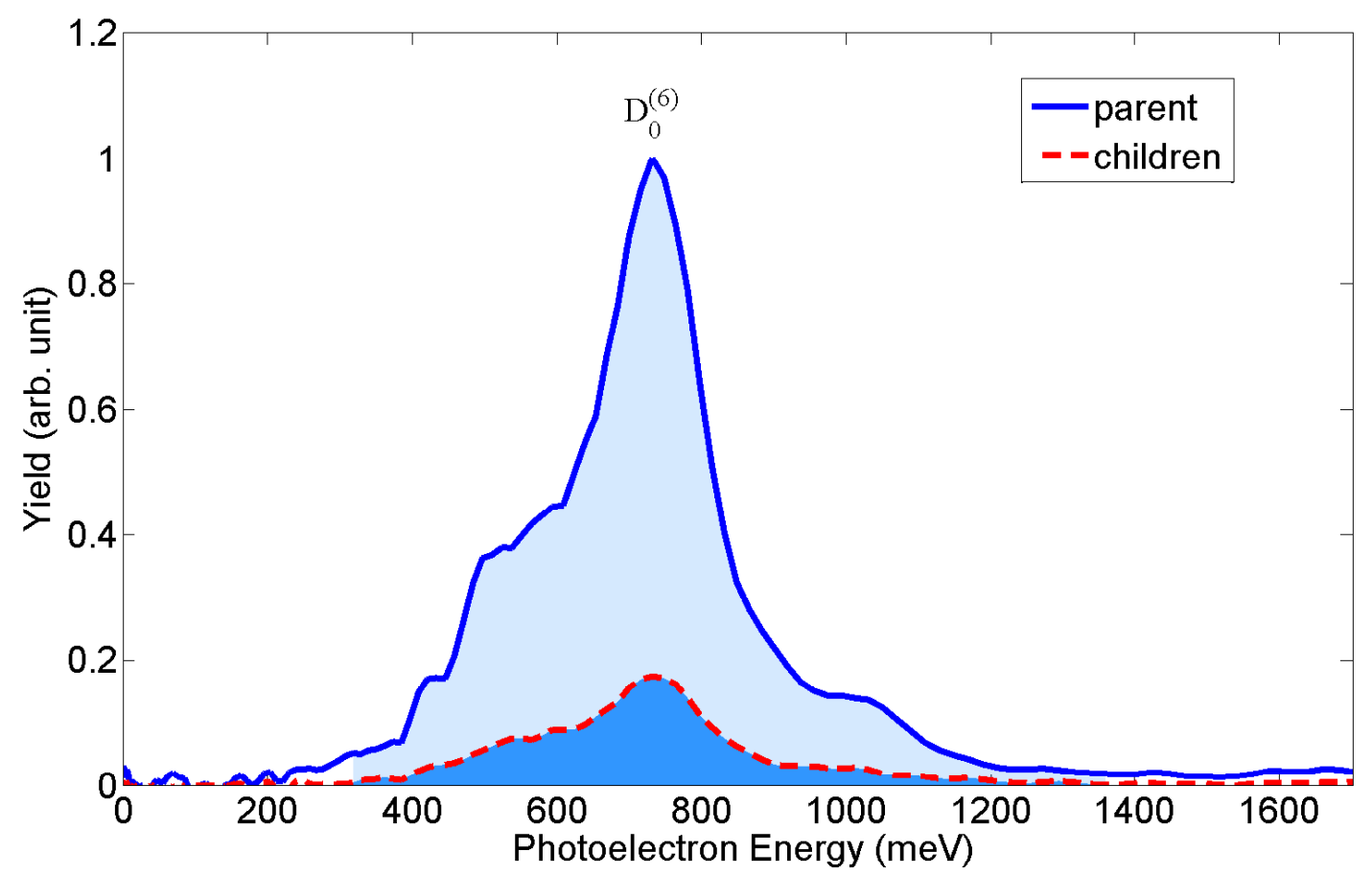

Figure A6: Photoelectron spectrum of $\mathrm{C}_{6} \mathrm{H}_{8}$.

No direct ionisation to dissociative states is observed.

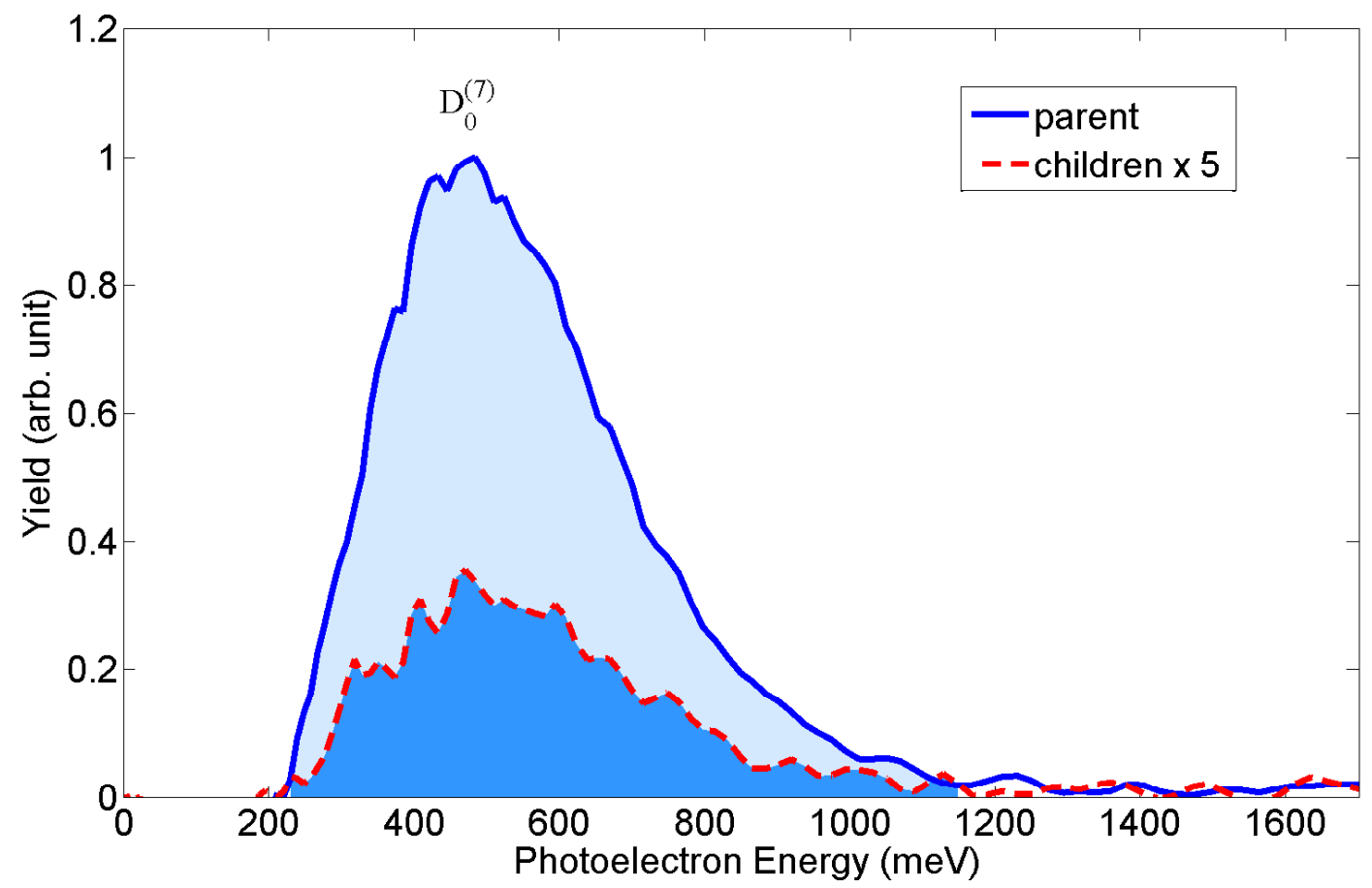

Figure A7: Photoelectron spectrum of $\mathrm{CS}_{2}$.

No direct ionisation to dissociative states is observed. The spectrum in coincidence with the fragment ions is multiplied by 5 for better viewing. 


\section{References}

[1] Pierre Agostini and Louis F DiMauro. The physics of attosecond light pulses. Reports on Progress in Physics, 67(6):813, 2004.

[2] Matthias F. Kling and Marc J.J. Vrakking. Attosecond electron dynamics. http://www. annualreviews.org/doi/abs/10.1146/annurev.physchem.59.032607.093532, 2008. PMID: 18031218.

[3] K. Kosma, S. A. Trushin, W. Fuß, and W. E. Schmid. Cyclohexadiene ring opening observed with 13 fs resolution: coherent oscillations confirm the reaction path. Phys. Chem. Chem. Phys., 11:172-181, 2009.

[4] Wen Li, Agnieszka A. Jaro-Becker, Craig W. Hogle, Vandana Sharma, Xibin Zhou, Andreas Becker, Henry C. Kapteyn, and Margaret M. Murnane. Visualizing electron rearrangement in space and time during the transition from a molecule to atoms. Proceedings of the National Academy of Sciences, 107(47):20219-20222, 2010.

[5] Spiridoula Matsika, Michael Spanner, Marija Kotur, and Thomas C. Weinacht. Ultrafast relaxation dynamics of uracil probed via strong field dissociative ionization. The Journal of Physical Chemistry A, 117(48):12796-12801, 2013.

[6] G. N. Gibson, R. R. Freeman, and T. J. McIlrath. Dynamics of the high-intensity multiphoton ionization of n2. Phys. Rev. Lett., 67:1230-1233, Sep 1991.

[7] H. Akagi, T. Otobe, A. Staudte, A. Shiner, F. Turner, R. Drner, D. M. Villeneuve, and P. B. Corkum. Laser tunnel ionization from multiple orbitals in hcl. Science, 325(5946):1364-1367, 2009.

[8] Andrey E. Boguslavskiy, Jochen Mikosch, Arjan Gijsbertsen, Michael Spanner, Serguei Patchkovskii, Niklas Gador, Marc J. J. Vrakking, and Albert Stolow. The multielectron ionization dynamics underlying attosecond strong-field spectroscopies. Science, 335(6074):1336$1340,2012$.

[9] Marija Kotur, Congyi Zhou, Spiridoula Matsika, Serguei Patchkovskii, Michael Spanner, and Thomas C. Weinacht. Neutral-ionic state correlations in strong-field molecular ionization. Phys. Rev. Lett., 109:203007, Nov 2012.

[10] Wen Li, Xibin Zhou, Robynne Lock, Serguei Patchkovskii, Albert Stolow, Henry C. Kapteyn, and Margaret M. Murnane. Time-resolved dynamics in n2o4 probed using high harmonic generation. Science, 322(5905):1207-1211, 2008.

[11] Hong Liu, Song-Feng Zhao, Min Li, Yongkai Deng, Chengyin Wu, Xiao-Xin Zhou, Qihuang Gong, and Yunquan Liu. Molecular-frame photoelectron angular distributions of strong-field tunneling from inner orbitals. Phys. Rev. A, 88:061401, Dec 2013.

[12] Brian K. McFarland, Joseph P. Farrell, Philip H. Bucksbaum, and Markus Ghr. High harmonic generation from multiple orbitals in n2. Science, 322(5905):1232-1235, 2008.

[13] Péter Sándor, Arthur Zhao, Tamás Rozgonyi, and Thomas Weinacht. Strong field molecular ionization to multiple ionic states: direct versus indirect pathways. Journal of Physics B: Atomic, Molecular and Optical Physics, 47(12):124021, 2014.

[14] Michael Spanner, Serguei Patchkovskii, Congyi Zhou, Spiridoula Matsika, Marija Kotur, and Thomas C. Weinacht. Dyson norms in xuv and strong-field ionization of polyatomics: Cytosine and uracil. Phys. Rev. A, 86:053406, Nov 2012.

[15] I. Znakovskaya, P. von den Hoff, S. Zherebtsov, A. Wirth, O. Herrwerth, M. J. J. Vrakking, R. de Vivie-Riedle, and M. F. Kling. Attosecond control of electron dynamics in carbon monoxide. Phys. Rev. Lett., 103:103002, Sep 2009.

[16] I. Znakovskaya, P. von den Hoff, N. Schirmel, G. Urbasch, S. Zherebtsov, B. Bergues, R. de VivieRiedle, K.-M. Weitzel, and M. F. Kling. Waveform control of orientation-dependent ionization of dcl in few-cycle laser fields. Phys. Chem. Chem. Phys., 13:8653-8658, 2011.

[17] J. A. Davies, J. E. LeClaire, R. E. Continetti, and C. C. Hayden. Femtosecond time-resolved photoelectronphotoion coincidence imaging studies of dissociation dynamics. The Journal of 
Chemical Physics, 111(1):1-4, 1999.

[18] C. Stefan Lehmann, N. Bhargava Ram, and Maurice H. M. Janssen. Velocity map photoelectronphotoion coincidence imaging on a single detector. Review of Scientific Instruments, 83(9):-, 2012.

[19] Arno Vredenborg, Wim G. Roeterdink, Cornelis A. de Lange, and Maurice H.M. Janssen. Revealing femtosecond multiphoton induced multichannel molecular ionization and fragmentation dynamics by photoelectronphotoion coincidence imaging. Chemical Physics Letters, 478(13):20 $-27,2009$.

[20] Andr T. J. B. Eppink and David H. Parker. Velocity map imaging of ions and electrons using electrostatic lenses: Application in photoelectron and photofragment ion imaging of molecular oxygen. Review of Scientific Instruments, 68(9), 1997.

[21] Tomislav Cvitas, Hans Gusten, and Leo Klasinc. Photoelectron spectra of lodobenzenes. J. Chem. Soc., Perkin Trans. 2, pages 962-965, 1977.

[22] N. A. Macleod, S. Wang, J. Hennessy, T. Ridley, K. P. Lawley, and R. J. Donovan. Ionic and rydberg states of cf3i studied by high resolution photoelectron (zeke-pfi) and resonance-enhanced multiphoton ionisation spectroscopy. J. Chem. Soc., Faraday Trans., 94:2689-2694, 1998.

[23] Leslie D. Waits, Ronald J. Horwitz, Robert G. Daniel, Joyce A. Guest, and Jeffrey R. Appling. Photofragmentation of cf3i+ produced by resonant multiphoton ionization. The Journal of Chemical Physics, 97(10), 1992.

[24] F. Aguirre and S. T. Pratt. Velocity map imaging of the photodissociation of cf3i+ in the $\mathrm{x}$ band. The Journal of Chemical Physics, 119(18), 2003.

[25] Wenzheng Fang, Lei Gong, Qiang Zhang, Xiaobin Shan, Fuyi Liu, Zhenya Wang, and Liusi Sheng. Dissociative photoionization of 1,3-butadiene: Experimental and theoretical insights. The Journal of Chemical Physics, 134(17):-, 2011.

[26] XiaoPeng Wang, ShengRui Tong, MaoFa Ge, WeiGang Wang, and DianXun Wang. Photoelectron spectroscopy of terpenoids and prediction of their rate. Chinese Science Bulletin, 55(35), 2010.

[27] T. S. Zyubina, A. M. Mebel, M. Hayashi, and S. H. Lin. Theoretical study of multiphoton ionization of cyclohexadienes and unimolecular decomposition of their mono- and dications. Phys. Chem. Chem. Phys., 10:2321-2331, 2008.

[28] A W Potts and G H Fattahallah. High-resolution ultraviolet photoelectron spectroscopy of co 2 , cos and cs 2. Journal of Physics B: Atomic and Molecular Physics, 13(13):2545, 1980.

[29] G.W. Fraser. The ion detection efficiency of microchannel plates (mcps). International Journal of Mass Spectrometry, 215(13):13 - 30, 2002. Detectors and the Measurement of Mass Spectra.

[30] N. Takahashi, Y. Adachi, M. Saito, and Y. Haruyama. Absolute detection efficiencies for kev energy atoms incident on a microchannel plate detector. Nuclear Instruments and Methods in Physics Research Section B: Beam Interactions with Materials and Atoms, 315(0):51 - 54, 2013. 25th International Conference on Atomic Collisions in Solids (ICACS-25).

[31] W. Fu, W. E. Schmid, and S. A. Trushin. Time-resolved dissociative intense-laser field ionization for probing dynamics: Femtosecond photochemical ring opening of 1,3-cyclohexadiene. The Journal of Chemical Physics, 112(19):8347-8362, 2000.

[32] Ryuji Itakura, Jun Watanabe, Akiyoshi Hishikawa, and Kaoru Yamanouchi. Ionization and fragmentation dynamics of benzene in intense laser fields by tandem mass spectroscopy. The Journal of Chemical Physics, 114(13):5598-5606, 2001.

[33] Hideo Harada, Seiji Shimizu, Tomoyuki Yatsuhashi, Shuji Sakabe, Yasukazu Izawa, and Nobuaki Nakashima. A key factor in parent and fragment ion formation on irradiation with an intense femtosecond laser pulse. Chemical Physics Letters, 342(56):563 - 570, 2001.

[34] Timothy Bohinski, Katharine Moore Tibbetts, Maryam Tarazkar, Dmitri Romanov, Spiridoula Matsika, and Robert J. Levis. Measurement of an electronic resonance in a ground-state, gas-phase acetophenone cation via strong-field mass spectrometry. The Journal of Physical Chemistry Letters, 4(10):1587-1591, 2013.

[35] Brett J. Pearson, Sarah R. Nichols, and Thomas C. Weinacht. Molecular fragmentation driven by 
ultrafast dynamic ionic resonances. Journal of Chemical Physics, 127:131101, 2007.

[36] Jesus Gonzalez-Vazquez, Leticia Gonzalez, Sarah R. Nichols, Thomas C. Weinacht, and Tamas Rozgonyi. Exploring wavepacket dynamics behind strong-field momentum-dependent photodissociation in ch2bri+. Phys. Chem. Chem. Phys., 12:14203-14216, 2010.

[37] Dominik Geißler, Tamás Rozgonyi, Jesús González-Vázquez, Leticia González, Philipp Marquetand, and Thomas C. Weinacht. Pulse-shape-dependent strong-field ionization viewed with velocity-map imaging. Phys. Rev. A, 84:053422, Nov 2011. 